\title{
Computational Modeling of Long Bone Microstructure and Ultrasonic Evaluation of the Fracture Healing Process
}

\author{
V. T. Potsika ${ }^{1}$, D. Polyzos ${ }^{2}$ and D. I. Fotiadis ${ }^{1,3, *}$ \\ ${ }^{1}$ Unit of Medical Technology and Intelligent Information Systems, Department of Materials \\ Science and Engineering, University of Ioannina, GR 45110 Ioannina, Greece \\ e-mail: vpotsika@cc.uoi.gr \\ ${ }^{2}$ Dept. of Mechanical Engineering and Aeronautics, University of Patras, GR 26500 Patras, \\ Greece \\ e-mail: polyzos@mech.upatras.gr \\ ${ }^{3}$ Foundation for Research and Technology Hellas, Institute of Molecular Biology and \\ Biotechnology, Dept. of Biomedical Research, GR 45110 Ioannina, Greece \\ e-mail: fotiadis@cc.uoi.gr \\ *corresponding author
}

\begin{abstract}
Bone is a composite medium organized into a complex hierarchical structure. Several research groups worldwide have applied ultrasonic techniques to investigate experimentally and numerically the structure of bone at different hierarchical levels. The advance in imaging modalities such as micro-computed tomography and scanning acoustic microscopy in combination with the use of robust computational tools has led to the development of realistic computational models of long bones. The ultrasonic assessment of bone microstructure and fracture healing using computational techniques can provide insight into complicated wave propagation effects which cannot be evaluated using traditional experimental procedures. This paper presents the milestone studies in the domain of ultrasonic evaluation of bone diseases such as osteoporosis and bone healing using numerical methods. The results indicate the significant diagnostic and monitoring role of quantitative ultrasound.
\end{abstract}

Keywords: long bones, fracture healing, osteoporosis, ultrasound

\section{Introduction}

Osteoporosis is characterized by reduced bone mass and disruption of bone architecture, resulting in increased risk of fragility fractures which represent the main clinical consequence of the disease (Hernlund et al. 2013). Osteoporosis is responsible for approximately 2 million fractures that occur annually, including hip, vertebral, wrist and other fractures (Dempster et al. 2011). Fragility fractures caused by the weakness of the skeleton due to osteoporosis are associated with substantial pain and suffering, disability and even death for affected patients and substantial costs to the society. Specifically, by 2025 the annual costs from osteoporosis are expected to reach approximately $\$ 25.3$ billion (Dempster et al. 2011). The main cause of osteoporosis is hormonal deficiency implying that the most frequent disease is post-menopausal 
osteoporosis (Laugier and Haïat 2011). According to the literature (Laugier and Haïat 2011), approximately $40 \%$ of post-menopausal women over the age of 50 and $15-30 \%$ of men will sustain one or more fragility fractures. Radiographic (X-ray) examination is the most common imaging modality applied for the diagnosis and monitoring of osteoporosis and bone healing in daily clinical practice. Although, it is a relative inexpensive method to diagnose the onset of bone diseases, the evaluation of the bone status may be subjective depending on the experience or clinical judgment of the orthopedic surgeon. Also, in about $5-10 \%$ of the incidence of fractures, complications may arise such as delayed unions and non-unions (Protopappas et al. 2008) which cannot be predicted using X-ray techniques as no quantitative information is provided. Therefore, the study of non-ionizing and non-invasive methods, which can provide both quantitative and qualitative information for bone assessment and enhance clinical decision making, is considered as an open research field.

To this end, quantitative ultrasound (QUS) is considered as a relative recent and promising method for the diagnosis and monitoring of osteoporosis and fracture healing. Specifically, there is increased interest during the last few decades in QUS techniques aiming to correlate ultrasonic propagation parameters with material and structural changes in pathological cases (Preininger et al. 2011, Machado et al. 2010, Casciaro et al. 2015, Barbieri et al. 2011). More recently, the use of numerical methods for the ultrasonic evaluation of bone diseases has provided supplementary information to experimental findings due to the rapid evolution of imaging modalities, such as micro-computed tomography ( $\mu$-CT) and scanning acoustic microscopy (SAM), and the availability of robust computational tools (Rohde et al. 2014, Potsika et al. 2014, Moilanen et al. 2007). One significant advantage of computational studies is that the role of the different types of ultrasonic waves can be investigated more easily and complications such as experimental artefacts can be avoided (Potsika et al. 2013).

The axial transmission, through transmission and the backscattering methods are the most popular ultrasonic techniques for bone characterization. Concerning the axial transmission method, a single or multiple emitters and receivers are used aligned along the long axis of the bone in contact with the skin or directly with the bone (Foiret et al. 2014). Measurements of the first arriving signal (FAS) velocity and attenuation as well as guided wave analysis have been performed to provide information for the evaluation of potential tissue defects not only in the periosteal region of bone, but also in deeper bone layers (Foiret et al. 2014, Kilappa et al. 2014, Potsika et al. 2014). The axial transmission method is mainly used to conduct measurements along the axis of long bones (i.e. radius, tibia). On the other hand, fewer experimental and numerical studies have used the through transmission and the backscattering methods. Concerning through transmission measurements, two transducers are placed in opposite bone directions (i.e. heel bone, distal radius) (Barbieri et al. 2011) and the parameters of interest are the FAS velocity and attenuation as well as the propagation of guided modes. Finally, the backscattering method uses a single transducer as an emitter and receiver and is applied in bone regions (i.e. the hip, spine) in which through-transmission measurements are difficult to be performed (Casciaro et al. 2015). The integrated reflection coefficient, the broadband ultrasound backscatter and apparent integrated backscatter (AIB), the time slope of apparent backscatter and the frequency slope of apparent backscatter are the main measured parameters (Liu et al. 2015, Karjalainen et al. 2012).

This study presents a comprehensive state of the art on computational modeling of long bone microstructure and ultrasonic evaluation of the fracture healing process. The significant diagnostic and monitoring potential of QUS is investigated in the evaluation of bone pathologies. Emphasis is given on: (a) the identification of the structural and material changes in cortical bone and callus tissue at different stages of fracture healing and osteoporosis, (b) the variation of porosity and the detection of pores larger than the size of a normal Haversian canal, (c) the frequency dependence of the examined parameters, (d) the application of different 
ultrasonic methods. The significance of numerical studies is shown and the parameters of interest are the FAS velocity, the propagation of guided waves and the scattering amplitude depending on the ultrasonic technique which is applied.

\section{Modeling and assessment of healthy and pathologic long bones}

\subsection{Computational Modeling of Intact and Osteoporotic Long Bones}

In the first numerical studies on ultrasonic characterization of intact and osteoporotic bones, simple 2D and 3D computational models of cortical bone were established (Nicholson et al. 2001, Bossy et al. 2002, Bossy et al. 2004). The diagnostic and monitoring potential of the FAS velocity and attenuation was investigated and the propagation of guided waves was introduced as a new method for the characterization of deeper cortical layers. More recently, the impact of the cortical microstructure on the FAS velocity was examined in (Rohde et al. 2014) and more realistic 2D numerical models were developed incorporating the cortical porosity based on SAM images. In (Vavva et al. 2009) the Mindlin Form II theory of gradient elasticity was used and intrinsic parameters were considered to correlate the bone's macrostructure with microstructure. According to these studies the microstructure of bone has a significant influence on the propagation of the FAS velocity and guided waves.

In (Potsika et al. 2016a, Potsika et al. 2017), we investigated the influence of cortical porosity on ultrasonic propagation features using the axial and the backscattering methods. More specifically, in (Potsika et al. 2016a) the axial transmission method was used and a parametric and a systematic numerical study was conducted to investigate the effect of alterations in cortical porosity and the occurrence of large basic multicellular units (BMUs) on the FAS velocity (Fig. 1). 2D geometries of cortical bone were established for various microstructural models mimicking normal and pathological tissue states based on SAM data. Table I presents the examined porosity scenarios. Emphasis was given on the identification of BMUs which may provoke the thinning of the cortical cortex and the increase of porosity at a later stage of osteoporosis.

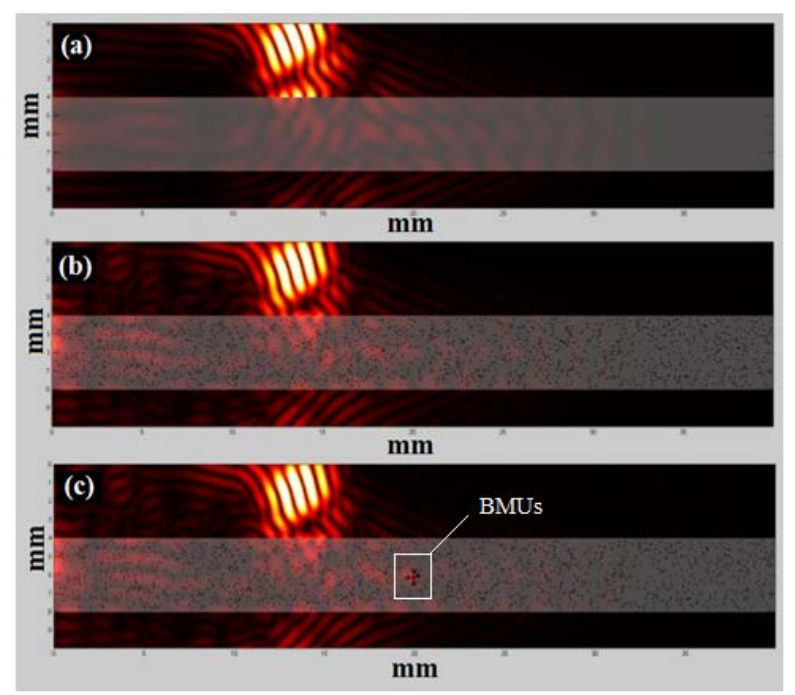

Fig. 1. Snapshots of axial ultrasonic propagation at $1 \mathrm{MHz}$ and time instant $10 \mu$ s for porosities:

(a) $0 \%$, (b) 5\%, (c) 5\% and 5 BMUs in the center of the plate (Potsika et al. 2016a). 


\begin{tabular}{llllll}
\hline Description & $\begin{array}{l}\text { Porosity } \\
{[\%]}\end{array}$ & $\begin{array}{l}\text { No. of } \\
\text { pores }\end{array}$ & $\begin{array}{l}\text { Pores' } \\
\text { radius } \\
{[\mu \mathrm{m}]}\end{array}$ & $\begin{array}{l}\text { No. of } \\
\text { RL/RLs }\end{array}$ & $\begin{array}{l}\text { Radius of } \\
\text { RL/RLs } \\
{[\mu \mathrm{m}]}\end{array}$ \\
\hline $\begin{array}{l}\text { Homogeneous bone } \\
\text { Porous bone, normal } \\
\text { pores }\end{array}$ & 5 & 0 & - & - & - \\
$\begin{array}{l}\text { Porous bone, normal } \\
\text { pores and 1 RL }\end{array}$ & 5 & 1592 & 40 & - & - \\
$\begin{array}{l}\text { Porous bone, normal } \\
\text { pores and 3 RLs }\end{array}$ & 5 & 1592 & 40 & 1 & 115 \\
$\begin{array}{l}\text { Porous bone, normal } \\
\text { pores and 5 RLs }\end{array}$ & 5 & 1592 & 40 & 3 & 115 \\
$\begin{array}{l}\text { Porous bone, normal } \\
\text { pores }\end{array}$ & 10 & 1592 & 60 & 5 & 115 \\
$\begin{array}{l}\text { Porous bone, normal } \\
\text { pores }\end{array}$ & 16 & 2263 & 60 & - & - \\
$\begin{array}{l}\text { Porous bone of } \\
\text { normal pores and RLs }\end{array}$ & 16 & 1400 & 60 & 235 & 115 \\
$\begin{array}{l}\text { Gradual distribution } \\
\text { of the pores, normal } \\
\text { pores and RLs }\end{array}$ & 16 & 1775 & $40,60,80$ & 192 & 115 \\
\hline
\end{tabular}

Table 1. Examined porosity scenarios.

The central excitation frequencies 0.5 and $1 \mathrm{MHz}$ were examined. The proposed configuration included one point source and multiple successive receivers in order to calculate the FAS velocity in small propagation paths and derive a velocity variation profile along the cortical surface. It was shown that: (a) the local FAS velocity decreases with increasing the porosity along the cortical cortex, (b) the local FAS velocity can capture porosity changes including the occurrence of BMUs with different number, size and depth of formation, and (c) the excitation frequency $0.5 \mathrm{MHz}$ is more sensitive for the assessment of cortical microstructure compared to the frequency of $1 \mathrm{MHz}$ depending on the relationship between the wavelength and the cortical thickness.

More recently, in (Potsika et al. 2017), the backscattering method was used to perform boundary element simulations of wave propagation in 2D numerical models of cortical bone with different porosities. Fig. 2, shows the examined porosity scenarios according to the description of Table I: (a) homogeneous bone (porosity $0 \%$ ), (b) porous bone with porosity 5\% and normal pores and (c) porous bone with porosity $10 \%$ and normal pores. The parameters of interest were the scattering amplitude and the acoustic pressure for various angles of propagation and receiving positions (Fig. 3). The evolution of the scattering effects was examined for the excitation frequencies $0.2,0.5$ and $1 \mathrm{MHz}$. The propagation of ultrasound in a composite medium such as bone stimulates multiple scattering mechanisms and a part of the incident energy is transferred to the pores. The excitation frequency and the structural features influence the amount of the scattered energy which is expressed by the amplitude of the scattering amplitude. It was found that for the propagation angle 180 degrees the scattering amplitude decreases with increasing the porosity and this was more prominent for higher excitation frequencies (Fig. 3). The results of this work revealed that the scattering amplitude and the acoustic pressure in the backward direction could provide significant information for the assessment of the cortical microstructure and early detect the onset of osteoporosis (Fig. 3). 


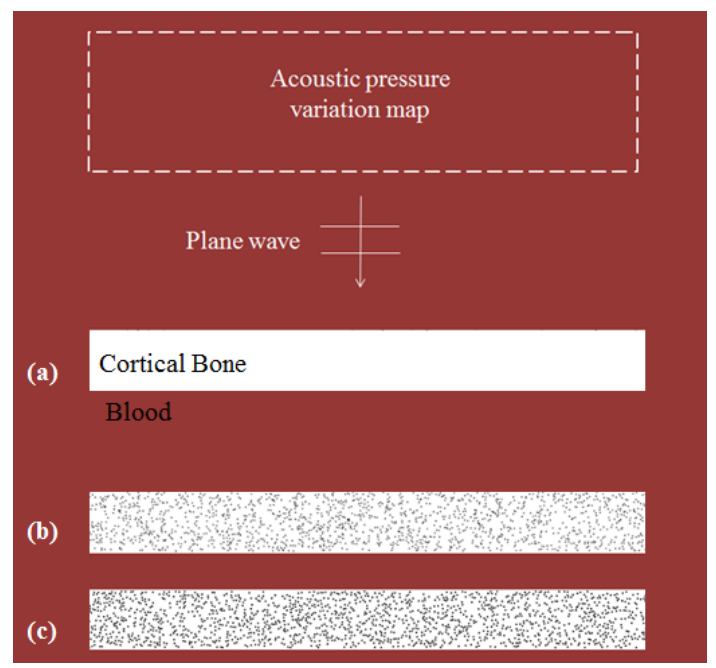

Fig. 2. Computational models of the porosity scenarios: (a) $0 \%$, (b) $5 \%$, and (c) $10 \%$. The ultrasonic configuration is also shown in (a) (Potsika et al. 2017).

\subsection{Computational Modeling of the Fracture Healing Process}

While the ultrasonic evaluation of intact and osteoporotic long bones using numerical methods has been extensively studied in the literature by several research groups, fewer studies have been presented for the numerical evaluation of the fracture healing process. In, (Dodd et al. 2007, Dodd et al. 2008, Protopappas et al. 2006), 2D isotropic models of healing long bones were presented in which the material properties of callus were varying to simulate different healing stages. The FAS velocity and attenuation were calculated using the axial transmission method. It was shown that the FAS velocity decreases in the first healing stages and gradually increases in later healing stages as the healing bone approaches the material and structural features of intact bone. However, the FAS velocity could not reveal changes in the callus tissue occurring within the endosteal region at successive healing stages. To this end, the propagation of guided waves was also examined based on the reassigned smoothed pseudo Wigner-Ville (RSPWV) energy distribution and the Lamb wave theory to derive the group velocity dispersion curves. It was shown that alterations in the callus tissue during fracture healing have a significant effect on the propagation of guided modes. In (Protopappas et al. 2007), a 3D finite element model of a healing long bone was presented and the FAS velocity and the propagation of guided waves were examined. Bone healing was simulated as a three stage process. The FAS velocity was found to decrease in the $1^{\text {st }}$ stage, remained the same in the $2^{\text {nd }}$ stage, and increased in the $3^{\text {rd }}$ stage. Nevertheless, when the FAS corresponded to a nondispersive lateral wave, its propagation velocity was almost unaffected by the elastic symmetry and geometry of the bone and could not characterize the callus tissue throughout its thickness (Protopappas et al. 2007). Also, it was shown that the irregularity and anisotropy of the bone as well as the material and geometrical changes that take place during the healing process have a significant impact on the propagation of guided waves.

More recently, we used SAM images to develop more realistic numerical models of healing long bones which account for the presence of callus porosity in successive healing weeks (Potsika et al. 2014). Ultrasonic wave propagation in healing long bones was simulated using an iterative effective medium approximation (IEMA) in order to account for different callus porosities (Aggelis et al. 2004). In Fig. 4, the SAM images from a sheep osteotomy model are 

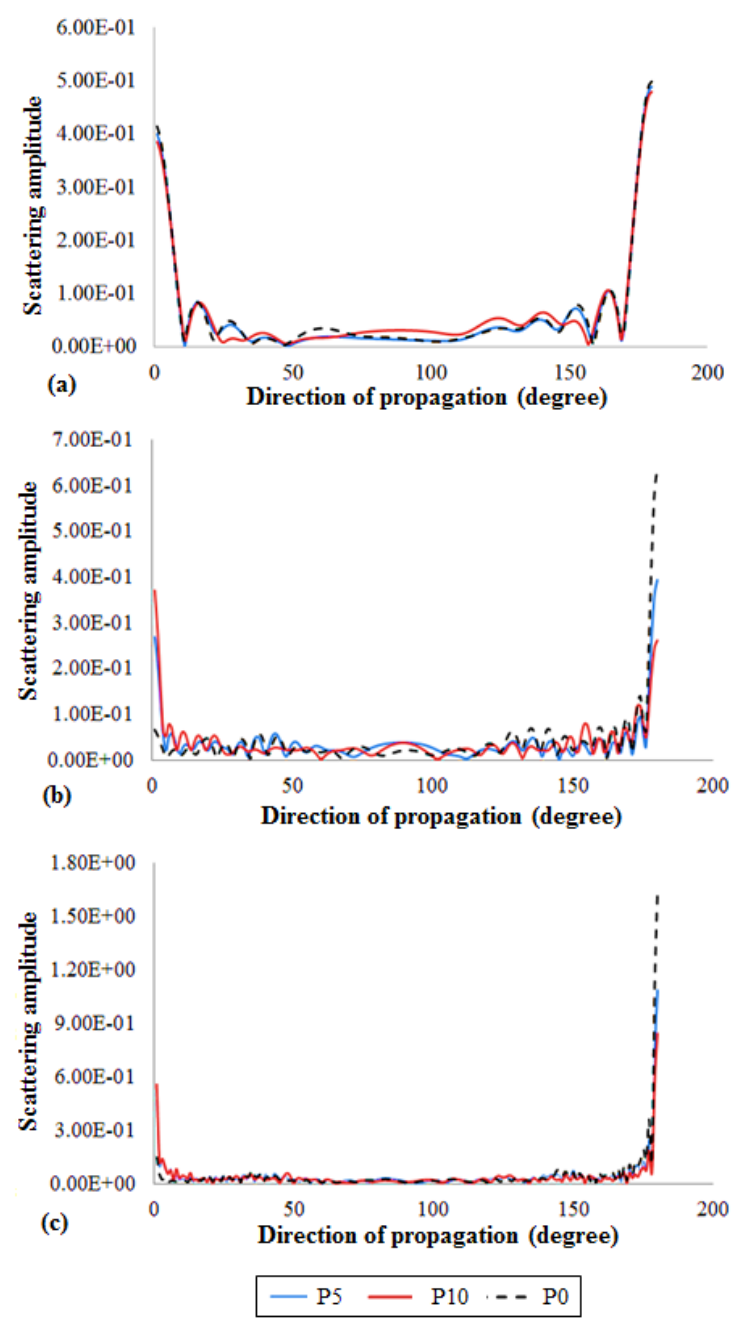

Fig. 3. Scattering amplitude evolution (in arbitrary units) for different propagation angles and excitation frequencies: (a) $0.2 \mathrm{MHz}$, (b) $0.5 \mathrm{MHz}$, and (c) $1 \mathrm{MHz}$ (Potsika et al. 2017).

illustrated representing the: (a) $2^{\text {nd }}$, (a) $3^{\text {rd }}$, (b) $6^{\text {th }}$ and (c) $9^{\text {th }}$ postoperative weeks (Preininger et al. 2011). The original material properties of cortical bone and callus as well as the callus porosity were derived using the SAM data. The excitation frequencies $0.3,0.5$ and $1 \mathrm{MHz}$ were examined. The effectiveness of IEMA in bone assessment was examined comparing the theoretical phase velocities with experimental measurements in cancellous bone mimicking phantoms. Guided wave analysis was performed for each healing stage and the results revealed that IEMA predictions could provide supplementary information for bone assessment during the healing process. Fig. 5 shows the guided wave analysis for the excitation frequency $1 \mathrm{MHz}$ of the signals obtained from: (a) the intact bone, (b) week 3, (c) week 6, and (d) week 9 after the osteotomy. It can be observed that in week 9, mode S1 was clearly identified when the Lamb curves were superimposed in the time-frequency diagrams indicating that the guided modes are gradually restored during the fracture healing process. 


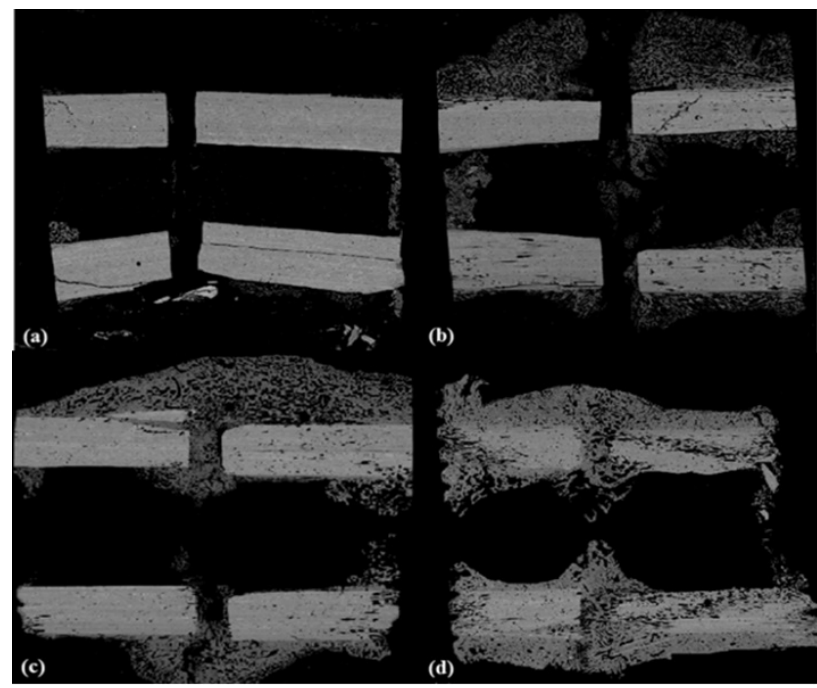

Fig. 4. SAM images from a sheep osteotomy model representing the: (a) $2^{\text {nd }}$ (a) $3^{\text {rd }}$, (b) $6^{\text {th }}$ and (c) $9^{\text {th }}$ postoperative week (Potsika et al. 2014).

In Potsika et al. (2016b) boundary element simulations of ultrasound propagation in healing long bones were performed to investigate the monitoring potential of backscattering parameters. More specifically, the interaction of a plane wave at $100 \mathrm{kHz}$ with healing long bones was examined by calculating the acoustic pressure and scattering amplitude in the backward direction. Callus was considered as a two-dimensional, non-homogeneous medium consisted of multiple layers with evolving material properties. Fig. 6 presents the computational models of healing long bones and the ultrasonic configuration considering multiple receiving positions. The cortical bone was considered immersed in blood to account for the role of soft tissues surrounding the bone.
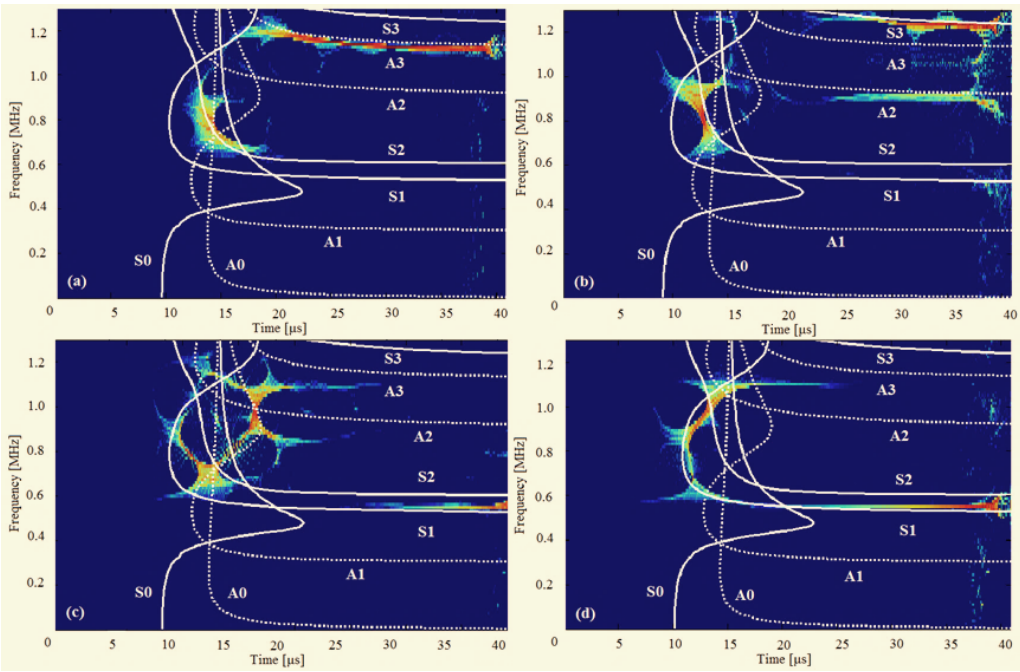

Fig. 5. Guided wave analysis for the excitation frequency of $1 \mathrm{MHz}$ of the signals obtained from: a) the intact bone, b) week 3, c) week 6, and d) week 9 after the osteotomy (Potsika et al. 2014). 


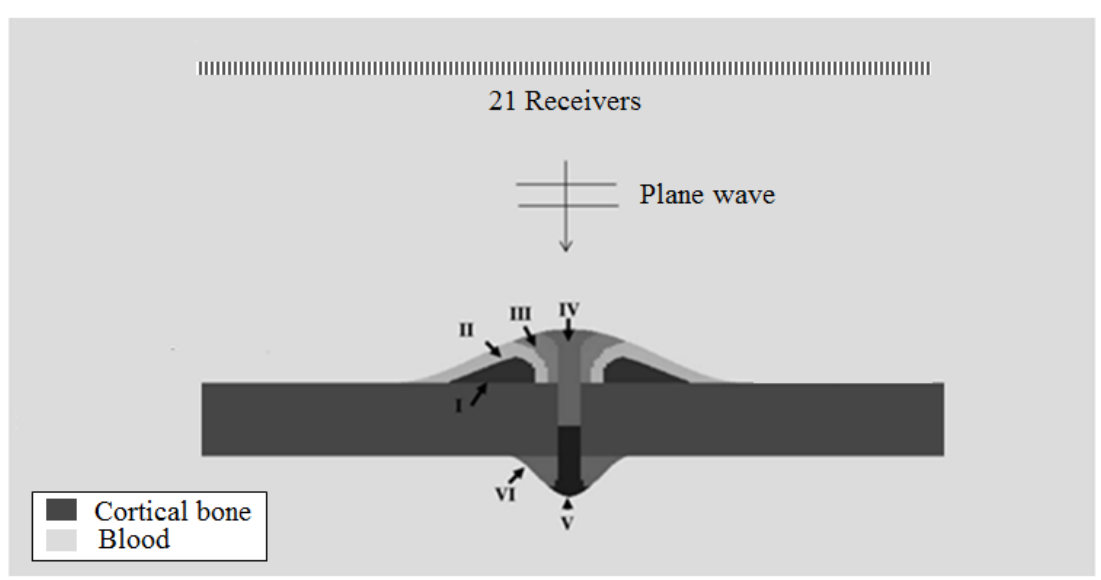

Fig. 6. Computational models of a healing long bone including the six different types of tissues involved in successive healing stages (Potsika et al. 2016b).

It was found that as fracture healing evolves the acoustic pressure curve gradually restores the values of intact bone. Specifically, in a region of $10 \mathrm{~mm}$ around the center of the callus the acoustic pressure was found to increase in the first healing stage and decrease in later healing stages. The opposite acoustic pressure behavior was observed for the receivers positioned at a distance larger than $10 \mathrm{~mm}$ from the center of the callus.

\section{Conclusions}

The ultrasonic evaluation of osteoporotic and healing long bones using numerical methods is a complex process due to the porous nature and hierarchical structure of bone. The advance in imaging modalities has enhanced the development of realistic numerical models which incorporate the inhomogeneities of the cortical and callus tissue. Also, different ultrasonic methods have been presented such as the axial, through and backscattering method and the selection of the proper method depends on several parameters such as the accessibility of the region of interest, the type of the pathology, the frequency and the measured parameters.

The axial transmission method is the most popular ultrasonic technique for bone characterization (Machado et al. 2010, Protopappas et al. 2007), while fewer numerical studies have used the through and the backscattering method (Potsika et. al 2017). The main estimated parameters are the FAS velocity and attenuation as well as the dispersion of guided waves. The Finite Difference and the Finite Element Method have been mainly applied, while the Boundary Element Method has been used to a more limited extent.

The FAS velocity was found to decrease during the first healing stages and gradually to increase in later healing stages approximating the values of intact bone (Protopappas et al. 2006). Moreover, the FAS velocity measured in small propagation paths was found to decrease with increasing the porosity reflecting changes in cortical porosity. Also, the FAS velocity was found to be an effective indicator for the identification of the occurrence of BMUs implying that it could predict osteoporosis at an early stage. However, this parameter is not sensitive to the evolution of the soft tissues which surround cortical bone, while according to the excitation frequency and the cortical thickness it may reflect changes only in the periosteal region. To this end, the study of guided waves has provided supplementary qualitative information for the evolution of callus and cortical tissue in deeper bone regions. Finally, the recent application of 
the backscattering method has shown that the scattering amplitude measured in the backward direction (180 degrees) decreases with increasing the cortical porosity. Also, the acoustic pressure measured in the backward direction for successive healing stages, was found to increase in the first healing stages and gradually decrease as the callus tissue is gradually restored.

Finally, the results of numerical studies have contributed significantly to the interpretation and validation of experimental and clinical findings. However, further experimental, clinical and numerical research work is necessary in order to better understand the complex attenuation phenomena induced by the occurrence of bone defects and incorporate QUS as a diagnostic and monitoring tool in daily clinical practice.

\section{References}

Aggelis D G, Tsinopoulos S V, Polyzos D (2004). An iterative effective medium approximation for wave dispersion and attenuation predictions in particulate composites, suspensions and emulsions, J. Acoust. Soc. Am., 9, 3443-3452.

Barbieri G, Barbieri C H, Mazzer B, Péla A (2011). Ultrasound Propagation Velocity and Broadband Attenuation Can Help Evaluate the Healing Process of an Experimental Fracture, J. Orthop. Res., 29, 444-451.

Bossy E, Talmant M, Laugier P (2002). Effect of bone cortical thickness on velocity measurements using ultrasonic axial transmission: a 2d simulation study, J. Acoust. Soc. Amer., 112, 297-307.

Bossy E, Talmant M, Laugier P (2004). Three-dimensional simulations of ultrasonic axial transmission velocity measurement on cortical bone models, J. Acoust. Soc. Amer., 115, 2314-2324.

Casciaro S, Conversano F, Pisani P, Muratore M (2015). New perspectives in echographic diagnosis of osteoporosis on hip and spine, Clin. Cases Min. \& Bone Metab., 12(2), 142150.

Dempster D (2011). Osteoporosis and the Burden of Osteoporosis-Related Fractures, Amer. J. Man. Care, 17, 164-169.

Dodd S P, Cunningham J L, Miles A W, Gheduzzi S, Humphrey V F (2008). Ultrasound transmission loss across transverse and oblique bone fractures: an in vitro study, Ultrasound in Med. \& Biol., 34(3), 454-462.

Dodd S P, Miles A W, Gheduzzi, Humphrey V F, Cunningham J L (2007). Modelling the effects of different fracture geometries and healing stages on ultrasound signal loss across a long bone fracture, Comp. Meth. Biomec. Biomed. Eng.,10(5), 371-375.

Foiret J, Minonzio J G, Chappard C, Talmant M, Laugier P (2014). Combined Estimation of Thickness and Velocities Using Ultrasound Guided Waves: A Pioneering Study on In Vitro Cortical Bone Samples, IEEE Trans. on Ultras., Ferroel., and Freq. control, 61(9), 14781488.

Hernlund E, Svedbom A, Ivergård M, Compston J, Cooper C, Stenmark J, McCloskey E V, Jönsson B, Kanis J A (2013). Osteoporosis in the European Union: medical management, epidemiology and economic burden, Arch Osteoporos, 8(1-2),136.

Karjalainen J P, Riekkinen O, Töyräs J, Hakulinen M, Kröger H, Rikkonen T, Salovaara K, Jurvelin J S (2012). Multi-site bone ultrasound measurements in elderly women with and without previous hip fractures, Osteoporos. Int., 23, 1287-1295.

Kilappa A, Xu K, Moilanen P, Heikolla E, Ta D, Timonen J (2013). Assessment of the fundamental flexular guided wave in cortical bone by an axial-transmission array transducer, Ultrasound in Med. \& Biol., 39(7), 1223-1232. 
Laugier P and Haïat G (2011). Bone quantitative ultrasound. Springer Dordrecht Heidelberg London New York: Science+Business Media B.V.

Liu C, Zhang R, Li Y, Xu F, Ta D, Wang W (2015). An Ultrasonic Backscatter Instrument for Cancellous Bone Evaluation in Neonates, Engineering, 1(3), 336-343.

Machado C B, de Albuquerque Pereira W C, Talmant M, Padilla F, Laugier P (2010). Computational evaluation of the compositional factors in fracture healing affecting ultrasound axial transmission measurements, Ultrasound in Med. \& Biol., 36(8), 13141326.

Moilanen P, Talmant M, Nicholson P H F, Cheng S, Timonen J, Laugier, P. (2007) Ultrasonically determined thickness of long cortical bones: Three-dimensional simulations of in vitro experiments, J. Acoust. Soc. Amer., 122, 2439-2445.

Nicholson P, Moilanen P, Kärkkäinen T, Timonen J, Cheng S (2002) Guided ultrasonic waves in long bones: modelling, experiment and application, Physiol. Meas., 23, 755-768.

Potsika V T, Grivas K N, Gortsas T, Protopappas V C, Polyzos D, Fotiadis D I (2017). Evaluation of ultrasonic scattering for different cortical bone porosities and excitation frequencies: a numerical study, Proc. 39th An. Intern. Conf. IEEE Engin. Med. Biol. Soc., Jeju Island, Korea.

Potsika V T, Grivas K N, Gortsas T, Iori G, Protopappas V C, Raum K, Polyzos D, Fotiadis D I (2016). Computational Study of the Effect of Cortical Porosity on Ultrasound Wave Propagation in Healthy and Osteoporotic Long Bones, Materials, 9(3), 1-21.

Potsika V T, Grivas K N, Gortsas T, Protopappas V C, Polyzos D, Fotiadis D I (2016). Boundary element simulation of ultrasonic backscattering during the fracture healing process, Proc. 38th An. Intern. Conf. IEEE Engin. Med. Biol. Soc., Orlando, Florida USA.

Potsika V T, Grivas K N, Protopappas V C, Vavva M G, Raum K, Rohrbach D, Polyzos D, Fotiadis D I (2014). Application of an effective medium theory for modeling ultrasound wave propagation in healing long bones, Ultrasonics, 54, 1219-1230.

Potsika V, Vavva M, Protopappas V, Polyzos D, Fotiadis D I (2013). Computational Modeling of Ultrasound Wave Propagation in Bone, Springer.

Preininger B, Checa S, Molnar F L, Fratzl P, Duda G N, Raum K (2011). Spatial-temporal mapping of bone structural and elastic properties in a sheep model following osteotomy, Ultrasound in Med. \& Biol., 37(3), 474-483.

Protopappas V C, Fotiadis D I, Malizos K N (2006). Guided ultrasound wave propagation in intact and healing long bones, Ultrasound in Med. \& Biol., 32(5), 693-708.

Protopappas V C, Kourtis I C, Kourtis L C, Malizos K N, Massalas C V, Fotiadis D I (2007). Three-dimensional finite element modeling of guided ultrasound wave propagation in intact and healing long bones, J. Acoust. Soc. Am., 121(6), 3907-3921.

Protopappas V C, Vavva M G, Fotiadis D I, Malizos K N (2008). Ultrasonic monitoring of bone fracture healing, IEEE Trans. Ultras., Ferroel., and Freq. control, 55(6), 1243-1255.

Rohde K, Rohrbach D, Gluer C C, Laugier P, Grimal Q, Raum K, Barkmann R (2014). Influence of Porosity, Pore Size, and Cortical Thickness on the Propagation of Ultrasonic Waves Guided Through the Femoral Neck Cortex: A Simulation Study, IEEE Trans. Ultras. Ferroel. Freq. Control, 61, 302-313.

Vavva M G, Protopappas V C, Gergidis L N, Charalampopoulos A, Fotiadis D I, Polyzos D (2009). Velocity dispersion of guided waves propagating in a free gradient elastic plate: Application to cortical, J. Acoust. Soc. Am., 125(5), 3414-3427. 